\title{
Well-Posedness and Stability for a Differential Problem with Hilfer-Hadamard Fractional Derivative
}

\author{
M. D. Kassim and N.-E. Tatar \\ Department of Mathematics and Statistics, King Fahd University of Petroleum and Minerals, Dhahran 31261, Saudi Arabia \\ Correspondence should be addressed to N.-E. Tatar; tatarn@kfupm.edu.sa
}

Received 5 June 2013; Accepted 24 October 2013

Academic Editor: Ademir Fernando Pazoto

Copyright (C) 2013 M. D. Kassim and N.-E. Tatar. This is an open access article distributed under the Creative Commons Attribution License, which permits unrestricted use, distribution, and reproduction in any medium, provided the original work is properly cited.

Motivated by the Hilfer fractional derivative (which interpolates the Riemann-Liouville derivative and the Caputo derivative), we consider a new type of fractional derivative (which interpolates the Hadamard derivative and its Caputo counterpart). We prove the well-posedness for a basic Cauchy type fractional differential equation involving this kind of derivative. This is established in an appropriate underlying space after proving the equivalence of this problem with a certain corresponding Volterra integral equation.

\section{Introduction}

In this work, we are concerned with the Hadamard derivative

$$
\begin{array}{r}
\left({ }_{H} \mathscr{D}_{a^{+}}^{\alpha} f\right)(x)=\frac{1}{\Gamma(1-\alpha)}\left(x \frac{d}{d x}\right) \int_{a}^{x}\left(\log \frac{x}{t}\right)^{-\alpha} \frac{f(t) d t}{t}, \\
a<x<b .
\end{array}
$$

Its Caputo counterpart is

$$
{ }_{H}^{c} \mathscr{D}_{a^{+}}^{\alpha} f=\mathscr{J}_{a^{+}}^{1-\alpha}\left(x \frac{d}{d x}\right) f
$$

where

$$
\left(\mathscr{J}_{a^{+}}^{\alpha} f\right)(x):=\frac{1}{\Gamma(\alpha)} \int_{a}^{x}\left(\log \frac{x}{t}\right)^{\alpha-1} \frac{f(t) d t}{t}, \quad a<x<b
$$

(see [1-5]). Here, we consider the following fractional derivative

$$
\left({ }_{H} \mathscr{D}_{a^{+}}^{\alpha, \beta} f\right)(x)=\left(\mathscr{J}_{a^{+}}^{\beta(1-\alpha)} \cdot{ }_{H} \mathscr{D}_{a^{+}}^{\alpha+\beta-\alpha \beta} f\right)(x) .
$$

This type of fractional derivative interpolates the Hadamard fractional derivative $(\beta=0)$ and the Caputo-Hadamard fractional derivative $(\beta=1)$. It has been introduced recently in [6]. In introducing this new fractional derivative we were motivated by the Hilfer fractional derivative of order $0<\alpha<$ 1 and type $0 \leq \beta \leq 1$ (see [7])

$$
\left(D_{0^{+}}^{\alpha, \beta} y\right)(x)=\left(I_{0^{+}}^{\beta(1-\alpha)} \frac{d}{d x} I_{0^{+}}^{(1-\beta)(1-\alpha)} f\right)(x),
$$

which interpolates the Riemann-Liouville derivative and the Caputo derivative.

We study the existence and uniqueness of solutions of a basic fractional differential equation

$$
\left({ }_{H} \mathscr{D}_{a^{+}}^{\alpha, \beta} y\right)(x)=f(x, y), \quad x>a>0
$$

with an appropriate initial condition in a suitable underlying space after proving the equivalence of this problem with a corresponding Volterra integral equation. In addition to that, we discuss the stability of solutions for a large and important class of nonlinearities. We find that solutions decay to zero at a logarithmic rate as time goes to infinity. To this end, we prove an inequality (which is important by itself).

The literature is very rich in works on well-posedness for fractional differential equations [8-17] (see also the books $[1,2,4,5]$ and the survey paper [18]) to cite but a few. The Hadamard fractional derivative may be found in the books $[1,2,4-6]$. Differential equations involving such a derivative and others have been treated in $[2,3]$. In contrast with the well-posedness, the stability issue and the long time behavior is not well studied $[6,12,19-23]$. 
The rest of the paper is organized as follows: the next section contains some material needed in our proofs. The different fractional derivatives as well as the new one are defined there. In Section 3, we present our problem and prove an existence and uniqueness result after establishing the equivalence of the differential problem with its corresponding integral equation. Section 4 is devoted to a stability result.

\section{Preliminaries}

In this section we present some definitions, lemmas, properties, and notation which will be used in our theorems later.

Definition 1 (see [2]). Let $\Omega=[a, b](0<a<b<\infty)$ be a finite interval and $0 \leq \gamma<1$, we introduce the weighted space $C_{\gamma, \log }[a, b]$ of continuous functions $g$ on $(a, b]$

$$
C_{\gamma, \log }[a, b]=\left\{g:(a, b] \longrightarrow \mathbf{R}:\left(\log \frac{x}{a}\right)^{\gamma} g(x) \in C[a, b]\right\} .
$$

In the space $C_{\gamma, \log }[a, b]$, we define the norm

$$
\|g\|_{C_{\gamma, \log }}=\left\|\left(\log \frac{x}{a}\right)^{\gamma} g(x)\right\|_{C}, \quad C_{0, \log }[a, b]=C[a, b] .
$$

Definition 2 (see [2]). Let $\delta=x(d / d x)$ be the $\delta$-derivative, for $n \in \mathbf{N}$ we denote by $C_{\delta, \gamma}^{n}[a, b](0 \leq \gamma<1)$ the Banach space of functions $g$ which have continuous $\delta$-derivatives on $[a, b]$ up to order $n-1$ and have the derivative $\delta^{n} g$ of order $n$ on $(a, b]$ such that $\delta^{n} g \in C_{\gamma, \log }[a, b]$

$$
\begin{aligned}
& C_{\delta, \gamma}^{n}[a, b] \\
& \quad=\left\{\delta^{k} g \in C[a, b], k=0, \ldots, n-1, \delta^{n} g \in C_{\gamma, \log }[a, b]\right\}
\end{aligned}
$$

with the norm

$$
\|g\|_{C_{\delta, \gamma}^{n}}=\sum_{k=0}^{n-1}\left\|\delta^{k} g\right\|_{C}+\left\|\delta^{n} g\right\|_{C_{\gamma, \log }} .
$$

When $n=0$, we set

$$
C_{\delta, \gamma}^{0}[a, b]=C_{\gamma, \log }[a, b] .
$$

Definition 3 (see [2]). Let $(a, b)(0 \leq a<b \leq \infty)$ be a finite or infinite interval of the half-axis $\mathbf{R}^{+}$and let $\alpha>0$. The Hadamard left-sided fractional integral $\mathscr{g}_{a^{+}}^{\alpha} f$ of order $\alpha>0$ is defined by

$$
\left(\mathcal{F}_{a^{+}}^{\alpha} f\right)(x):=\frac{1}{\Gamma(\alpha)} \int_{a}^{x}\left(\log \frac{x}{t}\right)^{\alpha-1} \frac{f(t) d t}{t}, \quad a<x<b,
$$

provided that the integral exists. When $\alpha=0$, we set

$$
\mathcal{F}_{a^{+}}^{0} f=f .
$$

Definition 4 (see [2]). Let $(a, b)(0 \leq a<b \leq \infty)$ be a finite or infinite interval of the half-axis $\mathbf{R}^{+}$and let $\alpha>0$. The Hadamard right-sided fractional integral $\mathscr{J}_{b^{-}}^{\alpha} f$ of order $\alpha>0$ is defined by

$$
\left(\mathscr{F}_{b^{-}}^{\alpha} f\right)(x):=\frac{1}{\Gamma(\alpha)} \int_{x}^{b}\left(\log \frac{t}{x}\right)^{\alpha-1} \frac{f(t) d t}{t}, \quad a<x<b,
$$

provided that the integral exists. When $\alpha=0$, we set

$$
\mathscr{J}_{b^{-}}^{0} f=f .
$$

Definition 5 (see [2]). The left-sided Hadamard fractional derivative of order $\alpha(0 \leq \alpha<1)$ on $(a, b)$ is defined by

$$
\left({ }_{H} \mathscr{D}_{a^{+}}^{\alpha} f\right)(x):=\delta\left(\mathcal{F}_{a^{+}}^{1-\alpha} f\right)(x) ;
$$

that is,

$$
\left({ }_{H} \mathscr{D}_{a^{+}}^{\alpha} f\right)(x)=\frac{1}{\Gamma(1-\alpha)}\left(x \frac{d}{d x}\right) \int_{a}^{x}\left(\log \frac{x}{t}\right)^{-\alpha} \frac{f(t) d t}{t},
$$

$$
a<x<b .
$$

In particular, when $\alpha=0$ we have

$$
{ }_{H} \mathscr{D}_{a^{+}}^{0} f=f .
$$

Definition 6 (see [2]). The right-sided Hadamard fractional derivative of order $\alpha(0 \leq \alpha<1)$ on $(a, b)$ is defined by

$$
\left({ }_{H} \mathscr{D}_{b^{-}}^{\alpha} f\right)(x):=-\delta\left(\mathcal{F}_{b^{-}}^{1-\alpha} f\right)(x) ;
$$

that is,

$$
\begin{array}{r}
\left({ }_{H} \mathscr{D}_{b^{-}}^{\alpha} f\right)(x)=-\left(x \frac{d}{d x}\right) \frac{1}{\Gamma(1-\alpha)} \int_{x}^{b}\left(\log \frac{t}{x}\right)^{-\alpha} \frac{f(t) d t}{t}, \\
a<x<b .
\end{array}
$$

In particular, when $\alpha=0$ we have

$$
{ }_{H} \mathscr{D}_{b^{-}}^{0} f=f .
$$

Definition 7. Let $(a, b)$ be a finite interval of the half-axis $\mathbf{R}^{+}$. The fractional derivative ${ }_{H}^{c} \mathscr{D}_{a^{+}}^{\alpha} f$ of order $\alpha(0<\alpha<1)$ on $(a, b)$ defined by

$$
{ }_{H}^{c} \mathscr{D}_{a^{+}}^{\alpha} f=\mathscr{J}_{a^{+}}^{1-\alpha} \delta f,
$$

where $\delta=x(d / d x)$, is called the Hadamard-Caputo fractional derivative of order $\alpha$.

In the rest of the paper we shall assume $a \neq 0$ when considering an interval $(a, b)$.

Lemma 8 (see [2]). Let $n \in \mathbf{N}_{0}=\{0,1, \ldots\}$ and let $\mu_{1}$ and $\mu_{2}$ be real numbers such that

$$
0 \leq \mu_{1} \leq \mu_{2}<1 .
$$


The following embeddings hold:

$$
C_{\delta}^{n}[a, b] \longrightarrow C_{\delta, \mu_{1}}^{n}[a, b] \longrightarrow C_{\delta, \mu_{2}}^{n}[a, b]
$$

with

$$
\begin{array}{r}
\|f\|_{C_{\delta, \mu_{2}}^{n}} \leq K_{\delta}\|f\|_{C_{\delta, \mu_{1}}^{n}}, \quad K_{\delta}=\min \left[1,\left(\log \frac{b}{a}\right)^{\mu_{2}-\mu_{1}}\right], \\
a \neq 0 .
\end{array}
$$

In particular,

$$
C[a, b] \longrightarrow C_{\mu_{1}, \log }[a, b] \longrightarrow C_{\mu_{2}, \log }[a, b],
$$

with

$$
\|f\|_{C_{\mu_{2}, \log }} \leq\left(\log \frac{b}{a}\right)^{\mu_{2}-\mu_{1}}\|f\|_{C_{\mu_{1}, \log }, \quad a \neq 0 .}
$$

Lemma 9 (see [2]). If $\alpha>0, \beta>0$ and $0<a<b<\infty$, then

$$
\begin{aligned}
& \left(\mathscr{F}_{a^{+}}^{\alpha}\left(\log \frac{t}{a}\right)^{\beta-1}\right)(x)=\frac{\Gamma(\beta)}{\Gamma(\alpha+\beta)}\left(\log \frac{x}{a}\right)^{\beta+\alpha-1}, \\
& \left(H^{D_{a^{+}}^{\alpha}}\left(\log \frac{t}{a}\right)^{\beta-1}\right)(x)=\frac{\Gamma(\beta)}{\Gamma(\beta-\alpha)}\left(\log \frac{x}{a}\right)^{\beta-\alpha-1} .
\end{aligned}
$$

In particular, if $\beta=1$ and $\alpha \geq 0$, then the Hadamard fractional derivative of a constant is not equal to zero:

$$
\left({ }_{H} \mathscr{D}_{a^{+}}^{\alpha} 1\right)(x)=\frac{1}{\Gamma(1-\alpha)}\left(\log \frac{x}{a}\right)^{-\alpha},
$$

when $0<\alpha<1$.

Lemma 10 (see [2]). Let $\alpha>0, \beta>0$ and $0 \leq \mu<1$. If $0<a<b<\infty$, then, for $f \in C_{\mu, \log }[a, b]$

$$
\mathscr{J}_{a^{+}}^{\alpha} \mathscr{J}_{a^{+}}^{\beta} f=\mathscr{J}_{a^{+}}^{\alpha+\beta} f
$$

holds at any point $x \in(a, b]$. When $f \in C[a, b]$ this relation is valid at any point $x \in[a, b]$.

Lemma 11 (see [2]). Let $0<\alpha \leq 1$ and $0<a<b<\infty$. The equality $\left(_{H^{2}} \mathscr{D}_{a^{+}}^{\alpha} y\right)(x)=0$ is valid for

$$
y(x)=c\left(\log \frac{x}{a}\right)^{\alpha-1}
$$

for any $c \in \mathbf{R}$.

Theorem 12 (see [2]). Let $0<\alpha<1$ and $0<a<b<\infty$. Also let $\mathscr{J}_{a^{+}}^{1-\alpha} f$ be the Hadamard fractional integral of order $1-\alpha$ of the function $f$.

$$
\text { If } f \in C_{\mu, \log }[a, b](0 \leq \mu<1) \text { and } \mathscr{F}_{a^{+}}^{1-\alpha} f \in C_{\delta, \mu}^{1}[a, b] \text {, }
$$

$$
\left(\mathscr{F}_{a^{+} H}^{\alpha} \mathscr{D}_{a^{+}}^{\alpha} f\right)(x)=f(x)-\frac{\left(\mathscr{F}_{a^{+}}^{1-\alpha} f\right)(a)}{\Gamma(\alpha)}\left(\log \frac{x}{a}\right)^{\alpha-1}
$$

holds at any point $x \in(a, b]$. If $f \in C[a, b]$ and $\mathcal{F}_{a^{+}}^{1-\alpha} f \in$ $C_{\delta}^{1}[a, b]$, then the relation holds at any point $x \in[a, b]$.
Lemma 13 (see [2]). Let $0<a<b<\infty, \alpha>0$ and $0 \leq \mu<$ 1.

(a) If $\mu>\alpha>0$, then the fractional integration operator $\mathscr{J}_{a^{+}}^{\alpha}$ is bounded from $C_{\mu, \log }[a, b]$ into $C_{\mu-\alpha, \log }[a, b]$ :

$$
\left\|\mathscr{F}_{a^{+}}^{\alpha} f\right\|_{C_{\mu-\alpha, \log }} \leq k_{1}\|f\|_{C_{\mu, \log }},
$$

where

$$
k_{1}=\left(\log \frac{b}{a}\right)^{\alpha} \frac{\Gamma(1-\mu)}{\Gamma[1+\alpha-\mu]} .
$$

In particular, $\mathcal{F}_{a^{+}}^{\alpha}$ is bounded in $C_{\mu, \log }[a, b]$.

(b) If $\mu \leq \alpha$, then the fractional integration operator $\mathcal{F}_{a^{+}}^{\alpha}$ is bounded from $C_{\mu, \log }[a, b]$ into $C[a, b]$ :

$$
\left\|\mathscr{J}_{a^{+}}^{\alpha} f\right\|_{C} \leq k_{2}\|f\|_{C_{\mu, \log }}
$$

where

$$
k_{2}=\left(\log \frac{b}{a}\right)^{\alpha-\mu} \frac{\Gamma(1-\mu)}{\Gamma(1+\alpha-\mu)} .
$$

In particular, $\mathcal{F}_{a^{+}}^{\alpha}$ is bounded in $C_{\mu, \log }[a, b]$.

Lemma 14 (see [2]). The Hadamard fractional integration operator $\mathcal{F}_{a^{+}}^{\alpha}$ of order $\alpha(\alpha>0)$ is a mapping from $C[a, b]$ to $C[a, b]$ and

$$
\left\|\mathscr{F}_{a^{+}}^{\alpha} g\right\|_{C[a, b]} \leq \frac{1}{\alpha \Gamma(\alpha)}\left(\log \frac{b}{a}\right)^{\alpha}\|g\|_{C[a, b]},
$$

where $g \in C[a, b]$.

Lemma 15 (see [17]). Let $0 \leq \gamma<1,0<a<c<b<\infty, g \in$ $C_{\gamma, \log }[a, c]$ and $g \in C[c, b]$. Then, $g \in C_{\gamma, \log }[a, b]$ and

$$
\|g\|_{C_{\gamma, \log }[a, b]} \leq \max \left\{\|g\|_{C_{\gamma, \log }[a, c]},\left(\log \frac{b}{a}\right)^{\gamma}\|g\|_{C[c, b]}\right\} .
$$

Lemma 16 (see [24]). Let $f, g$ be two continuous, positive functions defined on $\left[t_{0}, \infty\right), t_{0} \geq 0$, and $w:[0, \infty) \rightarrow[0, \infty)$ be a continuous monotonic nondecreasing function such that $w(0)=0$ and $w(x)>0$ for $x>0$. If $u$ is a positive differentiable function on $\left[t_{0}, \infty\right)$ that satisfies

$$
u^{\prime}(t) \leq f(t) w(u(t))+g(t), \quad t \in\left[t_{0}, \infty\right)
$$

then we have

$$
u(t) \leq W^{-1}\left[W\left(u\left(t_{0}\right)+\int_{t_{0}}^{t} g(s) d s\right)+\int_{t_{0}}^{t} f(s) d s\right],
$$

for the values oft, for which the right-hand side is well-defined, where

$$
W(r)=\int_{r_{0}}^{r} \frac{d s}{w(s)}, \quad r>r_{0}>0
$$


Theorem 17 (Banach fixed point theorem [2]). Let $(U, d)$ be a non-empty complete metric space, let $0 \leq w<1$, and let $T: U \rightarrow U$ be a map such that, for every $u, v \in U$, the relation

$$
d(T u, T v) \leq w d(u, v), \quad 0 \leq w<1
$$

holds. Then, the operator $T$ has a unique fixed point $u^{*} \in U$.

Furthermore, if $T^{k}(k \in \mathbf{N})$ is the sequence of operators defined by

$$
T^{1}=T, \quad T^{k}=T T^{k-1} k \in \mathbf{N} \backslash\{1\},
$$

then, for any $u_{0} \in U$, the sequence $\left\{T^{k} u_{0}\right\}_{k=1}^{\infty}$ converges to the above fixed point $u^{*}$.

Theorem 18 (Young's inequality). If $a$ and $b$ are nonnegative real numbers and $p$ and $q$ are positive real numbers such that $1 / p+1 / q=1$ then we have

$$
a b \leq \frac{a^{p}}{p}+\frac{b^{q}}{q} .
$$

Equality holds if and only if $a^{p}=b^{q}$.

Finally, we refer the reader to the nice treatments of Hadamard-type fractional calculus in $[25,26]$

\section{Existence and Uniqueness for an FDE with Hilfer-Hadamard Fractional Derivative}

In this section we discuss the existence, uniqueness and the stability of solutions of the Cauchy type problem (46) (below) with Hilfer-Hadamard fractional derivative.

Definition 19 (Hilfer-Hadamard fractional derivative (HHFD)). The left sided fractional derivative of order $\alpha$, $(0<\alpha<1)$ and type $0 \leq \beta \leq 1$ with respect to $x$ is defined by

$$
\left({ }_{H} \mathscr{D}_{a^{+}}^{\alpha, \beta} f\right)(x)=\left(\mathscr{g}_{a^{+}}^{\beta(1-\alpha)} \cdot{ }_{H} \mathscr{D}_{a^{+}}^{\alpha+\beta-\alpha \beta} f\right)(x)
$$

for functions for which the expression on the right hand side exists, where ${ }_{H} \mathscr{D}_{a^{+}}^{\alpha+\beta-\alpha \beta}$ is the Hadamard fractional derivative (Definition 5).

This new fractional derivative (introduced for the first time in [6]) may be viewed as interpolating the Hadamard fractional derivative and the Hadamard-Caputo fractional derivative. Indeed for $\beta=0$ this derivative (46) reduces to the Hadamard fractional derivative (Definition 5) and when $\beta=1$, we recover the Hadamard-Caputo fractional derivative (Definition 7).

We will study the existence and uniqueness for the Cauchy type problem

$$
\begin{gathered}
\left({ }_{H} \mathscr{D}_{a^{+}}^{\alpha, \beta} y\right)(x)=f(x, y(x)), \quad x>a>0 \\
\left(\mathscr{J}_{a^{+}}^{(1-\beta)(1-\alpha)} y\right)(a)=c .
\end{gathered}
$$

We consider the underlying spaces defined by

$$
\begin{aligned}
& C_{1-\gamma, \mu}^{\alpha, \beta}[a, b] \\
& \quad=\left\{y \in C_{1-\gamma, \log }[a, b],{ }_{H} \mathscr{D}_{a^{+}}^{\alpha, \beta} y \in C_{\mu, \log }[a, b]\right\}, \\
& C_{1-\gamma, \log }^{\gamma}[a, b] \\
& \quad=\left\{y \in C_{1-\gamma, \log }[a, b],{ }_{H} \mathscr{D}_{a^{+}}^{\gamma} y \in C_{1-\gamma, \log }[a, b]\right\},
\end{aligned}
$$

where $\gamma=\alpha+\beta-\alpha \beta$ and $0 \leq \mu<1$. It is clear that $0<\gamma<1$ for $0<\alpha, \beta<1$.

Here, $C_{1-\gamma, \log }[a, b]$ and $C_{\mu, \log }[a, b]$ are weighted spaces of continuous functions on $(a, b]$ defined by

$$
C_{\gamma, \log }[a, b]=\left\{g:(a, b] \longrightarrow \mathbf{R}:\left(\log \frac{x}{a}\right)^{\gamma} g(x) \in C[a, b]\right\} .
$$

Our investigations are based on reducing the fractional differential problem to a Volterra integral equation of the second kind:

$$
\begin{aligned}
y(x)= & \frac{c}{\Gamma(\gamma)}\left(\log \frac{x}{a}\right)^{\gamma-1}+\frac{1}{\Gamma(\alpha)} \\
& \times \int_{a}^{x}\left(\log \frac{x}{t}\right)^{\alpha-1} f[t, y(t)] \frac{d t}{t}, \quad x>a,
\end{aligned}
$$

and then using the Banach fixed point theorem.

3.1. Equivalence of the Cauchy Type Problem and the Volterra Integral Equation. Here, we prove the equivalence of the Cauchy type problem (46) and the nonlinear Volterra integral equation (50) in the sense that, if $y \in C_{1-\gamma, \log }^{\gamma}[a, b]$ satisfies one of them, then it also satisfies the other one. To establish this result, we assume that the function $f[\cdot, y(\cdot)]$ belongs to $C_{\mu, \log }[a, b]$ for any $y \in C_{\mu, \log }[a, b]$. We need the following lemma.

Lemma 20. Let $0<a<b<\infty, \alpha>0,0 \leq \mu<1$ and $g \in C_{\mu, \log }[a, b]$. If $\alpha>\mu$, then $\mathscr{J}_{a^{+}}^{\alpha} g$ is continuous on $[a, b]$ and

$$
\mathcal{F}_{a^{+}}^{\alpha} g(a)=\lim _{x \rightarrow a^{+}} \mathcal{J}_{a^{+}}^{\alpha} g(x)=0 .
$$

Proof. Since $g \in C_{\mu, \log }[a, b]$ then $(\log (x / a))^{\mu} g(x)$ is continuous on $[a, b]$ and on $[a, b]$ we have

$$
\left|\left(\log \frac{x}{a}\right)^{\mu} g(x)\right| \leq M
$$

for some positive constant $M$. Therefore,

$$
\left|\left(\mathscr{F}_{a+}^{\alpha} g\right)(x)\right| \leq M\left(\mathscr{F}_{a+}^{\alpha}\left(\log \frac{t}{a}\right)^{-\mu}\right)(x),
$$

and by using Lemma 9 (with $\beta=1-\mu>0$ ) we have

$$
\left|\left(\mathscr{J}_{a+}^{\alpha} g\right)(x)\right| \leq M \frac{\Gamma(1-\mu)}{\Gamma(\alpha+1-\mu)}\left(\log \frac{x}{a}\right)^{\alpha-\mu} .
$$

As $\alpha>\mu$, we obtain the result. 
Theorem 21. Let $\gamma=\alpha+\beta-\alpha \beta$ where $0<\alpha<1$ and $0 \leq \beta \leq$ 1. Let $f:(a, b] \times \mathbf{R} \rightarrow \mathbf{R}$ be a function such that $f(\cdot, y(\cdot)) \in$ $C_{\mu, \log }[a, b]$ for any $y \in C_{\mu, \log }[a, b]$ with $1-\gamma \leq \mu<1-\beta(1-\alpha)$.

If $y \in C_{1-\gamma, \log }^{\gamma}[a, b]$, then $y$ satisfies the (CFDP) (46) if and only if $y$ satisfies the (IE) (50).

Proof. First we prove the necessity. Let $y \in C_{1-\gamma, \log }^{\gamma}[a, b]$ be a solution of problem (46). We want to prove that $y$ is also a solution of the integral equation (50). By the definition of the space $C_{1-\gamma, \log }^{\gamma}[a, b]$ (relation (48)) we have

$$
\delta\left(\mathcal{F}_{a^{+}}^{1-\gamma} y\right)={ }_{H} \mathscr{D}_{a^{+}}^{\gamma} y \in C_{1-\gamma, \log }[a, b] .
$$

Moreover, by Lemma 13(b) we have $\mathscr{J}_{a^{+}}^{1-\gamma} y \in C[a, b]$ since $y \in C_{1-\gamma, \log }[a, b]$. Then, by Definition 2 , we have

$$
\mathcal{J}_{a^{+}}^{1-\gamma} y \in C_{\delta, 1-\gamma}^{1}[a, b] .
$$

Thus, we can apply Theorem 12 (with $f$ replaced by $y$ ) to get

$$
\begin{array}{r}
\left(\mathscr{J}_{a^{+} H^{\gamma}}^{\gamma} \mathscr{D}_{a^{+}}^{\gamma} y\right)(x)=y(x)-\frac{\left(\mathscr{J}_{a^{+}}^{1-\gamma} y\right)(a)}{\Gamma(\gamma)}\left(\log \frac{x}{a}\right)^{\gamma-1}, \\
x \in(a, b]
\end{array}
$$

or

$$
\left(\mathscr{F}_{a^{+} H^{\gamma}}^{\gamma} \mathscr{D}_{a^{+}}^{\gamma} y\right)(x)=y(x)-\frac{c}{\Gamma(\gamma)}\left(\log \frac{x}{a}\right)^{\gamma-1}, \quad x \in(a, b],
$$

where $c$ comes from the initial condition in (46). By our hypothesis $f[\cdot, y(\cdot)] \in C_{\mu, \log }[a, b]$, since $y \in C_{1-\gamma, \log }[a, b] \subset$ $C_{\mu, \log }[a, b]$, Lemma 13(a) and (b) we see that the integral $\mathscr{J}_{a^{+}}^{\alpha} f[\cdot, y(\cdot)] \in C_{\mu-\alpha, \log }[a, b]$ for $\mu>\alpha$ and $\mathscr{F}_{a^{+}}^{\alpha} f[\cdot, y(\cdot)] \epsilon$ $C[a, b]$ for $\mu \leq \alpha$. Applying the operator $\mathcal{F}_{a^{+}}^{\alpha}$ to both sides of (46) we get

$$
\mathscr{F}_{a^{+}}^{\alpha} \mathcal{F}_{a^{+}}^{\beta(1-\alpha)}\left({ }_{H} \mathscr{D}_{a^{+}}^{\gamma} y\right)(x)=\mathcal{F}_{a^{+}}^{\alpha} f[x, y(x)], \quad x \in(a, b] .
$$

We can sum up the exponents by Lemma 10 to get

$$
\mathscr{J}_{a^{+} H}^{\alpha+\beta(1-\alpha)} \mathscr{D}_{a^{+}}^{\gamma} y(x)=\mathscr{J}_{a^{+}}^{\alpha} f[x, y(x)], \quad x \in(a, b],
$$

or

$$
\left(\mathscr{J}_{a^{+} H}^{\gamma} \mathscr{D}_{a^{+}}^{\gamma} y\right)(x)=\left(\mathscr{F}_{a^{+}}^{\alpha} f[t, y(t)]\right)(x), \quad x \in(a, b] .
$$

From (58) and (61) we obtain

$$
y(x)=\frac{c}{\Gamma(\gamma)}\left(\log \frac{x}{a}\right)^{\gamma-1}+\left(\mathscr{J}_{a^{+}}^{\alpha} f[t, y(t)]\right)(x),
$$

which is (50), and hence the necessity is proved.

Now, we prove the sufficiency. Let $y \in C_{1-\gamma, \log }^{\gamma}[a, b]$ satisfy (50), then ${ }_{H^{2}} \mathscr{D}_{a^{+}}^{\gamma} y$ exists and ${ }_{H^{\prime}} \mathscr{D}_{a^{+}}^{\gamma} y \in C_{1-\gamma, \log }[a, b]$.
Applying the operator ${ }_{H} \mathscr{D}_{a^{+}}^{\gamma}$ to both sides of the last identity we find

$$
\begin{aligned}
\left({ }_{H} \mathscr{D}_{a^{+}}^{\gamma} y\right)(x)= & \frac{c}{\Gamma(\gamma)} H^{\mathscr{D}_{a^{+}}^{\gamma}}\left(\log \frac{t}{a}\right)^{\gamma-1}(x) \\
& +\left({ }_{H} \mathscr{D}_{a^{+}}^{\gamma} \mathcal{F}_{a^{+}}^{\alpha} f[t, y(t)]\right)(x) .
\end{aligned}
$$

By using Lemma 10, Definition 5, Lemma 23 and the hypothesis $f[\cdot, y(\cdot)] \in C_{\mu, \log }[a, b]$, we have

$$
\begin{aligned}
\left({ }_{H} \mathscr{D}_{a^{+}}^{\gamma} y\right)(x) & =\delta\left(\mathscr{J}_{a^{+}}^{1-\gamma} \mathscr{J}_{a^{+}}^{\alpha} f[t, y(t)]\right)(x) \\
& =\delta\left(\mathscr{J}_{a^{+}}^{1-\beta(1-\alpha)} f[t, y(t)]\right)(x) \\
& =\left({ }_{H} \mathscr{D}_{a^{+}}^{\beta(1-\alpha)} f[t, y(t)]\right)(x), \quad x \in(a, b] .
\end{aligned}
$$

From (64) and the fact that ${ }_{H} \mathscr{D}_{a^{+}}^{\gamma} y \in C_{1-\gamma, \log }[a, b]$, we obtain that

$$
H^{\mathscr{D}_{a^{+}}^{\beta(1-\alpha)}} f[\cdot, y(\cdot)] \in C_{1-\gamma, \log }[a, b] .
$$

Next, applying the operator $\mathscr{J}_{a^{+}}^{\beta(1-\alpha)}$ to both sides of (64) we get

$$
\left(\mathscr{J}_{a^{+} H}^{\beta(1-\alpha)} \mathscr{D}_{a^{+}}^{\gamma} y\right)(x)=\left(\mathscr{J}_{a^{+} H}^{\beta(1-\alpha)} \mathscr{D}_{a^{+}}^{\beta(1-\alpha)} f[t, y(t)]\right)(x)
$$

that is,

$$
\mathscr{J}_{a^{+}}^{\beta(1-\alpha)} \delta\left(\mathscr{J}_{a^{+}}^{1-\gamma} y\right)(x)=\left(\mathscr{J}_{a^{+} H}^{\beta(1-\alpha)} \mathscr{D}_{a^{+}}^{\beta(1-\alpha)} f[t, y(t)]\right)(x) .
$$

By virtue of

$$
\begin{aligned}
\delta & \left(\mathcal{J}_{a^{+}}^{1-\beta(1-\alpha)} f[t, y(t)]\right) \\
& ={ }_{H} \mathscr{D}_{a^{+}}^{\beta(1-\alpha)} f[\cdot, y(\cdot)] \in C_{1-\gamma, \log }[a, b],
\end{aligned}
$$

and $\gamma>\beta(1-\alpha)$ and Definition 2, we have $\mathscr{J}_{a^{+}}^{1-\beta(1-\alpha)} f \in$ $C_{\delta ; 1-\gamma}^{1}[a, b]$ (see the first part of the proof, or Lemma 13(b), for the continuity of $g_{a^{+}}^{1-\beta(1-\alpha)} f$ for $\left.\mu<1-\beta(1-\alpha)\right)$. Then, Theorem 12 allows us to write

$$
\begin{aligned}
\left({ }_{H} \mathscr{D}_{a^{+}}^{\alpha, \beta} y\right)(x)= & f(x, y)-\frac{\left(\mathcal{F}_{a^{+}}^{1-\beta(1-\alpha)} f\right)(a)}{\Gamma[\beta(1-\alpha)]} \\
& \times\left(\log \frac{x}{a}\right)^{\beta(1-\alpha)-1}, \quad x \in(a, b] .
\end{aligned}
$$

Lemma 20 implies that

$$
\left(\mathscr{J}_{a^{+}}^{1-\beta(1-\alpha)} f\right)(a)=0
$$

because $1-\beta(1-\alpha)>\mu$. Hence, the relation (69) reduces to

$$
\left({ }_{H} \mathscr{D}_{a^{+}}^{\alpha, \beta} y\right)(x)=f(x, y(x)), \quad x>a .
$$


Now, we show that the initial condition in (46) also holds. To this end we apply the operator $\mathcal{J}_{a^{+}}^{1-\gamma}$ to both sides of (50):

$$
\begin{aligned}
\left(\mathscr{F}_{a^{+}}^{1-\gamma} y\right)(x)= & \frac{c}{\Gamma(\gamma)} \mathscr{J}_{a^{+}}^{1-\gamma}\left(\log \frac{t}{a}\right)^{\gamma-1}(x) \\
& +\left(\mathscr{F}_{a^{+}}^{1-\gamma} \mathcal{J}_{a^{+}}^{\alpha} f[t, y(t)]\right)(x)
\end{aligned}
$$

and use the Lemma 8 (with $\alpha$ replaced by $1-\gamma$ and $\beta$ by $\gamma$ ) and the Lemma 9 to obtain

$$
\left(\mathscr{J}_{a^{+}}^{1-\gamma} y\right)(x)=c+\left(\mathscr{F}_{a^{+}}^{1-\beta(1-\alpha)} f[t, y(t)]\right)(x) .
$$

In (73), taking the limit as $x \rightarrow a$, we obtain

$$
\left(\mathscr{J}_{a^{+}}^{1-\gamma} y\right)(a)=c
$$

mentioned above $\left.\mathscr{J}_{a^{+}}^{1-\beta(1-\alpha)} f[t, y(t)](a)=0\right)$. Therefore, the sufficiency is proved, which completes the proof of Theorem 21.

3.2. Existence and Uniqueness of a Solution. In this section we establish the existence of a unique solution to the Cauchy type problem (46) in the space $C_{1-\gamma, \mu}^{\alpha, \beta}[a, b]$ defined in (47) above under the conditions of Theorem 21 and an additional Lipschitz condition

$$
\left\|f\left[x, y_{1}\right]-f\left[x, y_{2}\right]\right\|_{C_{1-\gamma, \log }[a, b]} \leq A\left\|y_{1}-y_{2}\right\|_{C_{1-\gamma, \log }[a, b]}
$$

for some positive constant $A$ and every $x \in[a, b]$.

Theorem 22. Let $\gamma=\alpha+\beta-\alpha \beta$ where $(0<\alpha<1,0 \leq \beta \leq 1)$. Assume that $f:(a, b] \times \mathbf{R} \rightarrow \mathbf{R}(a>0)$ is a function such that $f[\cdot, y(\cdot)] \in C_{\mu, \log }[a, b]$ for any $y \in C_{\mu, \log }[a, b]$ with $1-\gamma \leq$ $\mu<1-\beta(1-\alpha)$ and is Lipschitz continuous with respect to its second variable. Then, there exists a unique solution $y$ for the Cauchy type problem (46) in the space $C_{1-\gamma, \mu}^{\alpha, \beta}[a, b]$.

Proof. First we prove the existence of a unique solution $y$ in the space $C_{1-\gamma, \log }[a, b]$. According to Theorem 21, it suffices to prove the existence of a unique solution $y \in C_{1-\gamma, \log }[a, b]$ to the nonlinear Volterra integral equation (50).

Let us select $x_{1}$ in $(a, b)$ such that

$$
w_{1}:=\frac{A \Gamma(\gamma)}{\Gamma(\alpha+\gamma)}\left(\log \frac{x_{1}}{a}\right)^{2 \alpha}<1,
$$

where $A>0$ is the Lipschitz constant. We start by proving that a unique solution $y \in C_{1-\gamma, \log }\left[a, x_{1}\right]$ to (50) exists on the interval $\left(a, x_{1}\right]$. It is easy to see that the space $C_{1-\gamma, \log }\left[a, x_{1}\right]$ is a complete metric space when equipped with the distance given by

$$
\begin{aligned}
d\left(y_{1}, y_{2}\right) & =\left\|y_{1}-y_{2}\right\|_{C_{1-\gamma, \log }\left[a, x_{1}\right]} \\
& :=\max _{x \in\left[a, x_{1}\right]}\left|\left(\log \frac{x}{a}\right)^{1-\gamma}\left[y_{1}(x)-y_{2}(x)\right]\right| .
\end{aligned}
$$

The integral equation (50) takes the form

$$
y(x)=(T y)(x)
$$

where

$$
(T y)(x)=y_{0}(x)+\left(\mathscr{F}_{a^{+}}^{\alpha} f[t, y(t)]\right)(x)
$$

with

$$
y_{0}(x)=\frac{c}{\Gamma(\gamma)}\left(\log \frac{x}{a}\right)^{\gamma-1} .
$$

We claim that $T$ maps $C_{1-\gamma, \log }\left[a, x_{1}\right]$ into itself. Indeed, $y_{0}$ given by (80) is clearly in $C_{1-\gamma, \log }\left[a, x_{1}\right]$. Also, since $f[\cdot, y] \in$ $C_{\mu, \log }[a, b]$ for any $y \in C_{\mu, \log }[a, b]$ with $\mu \in \mathbf{R}(0 \leq \mu<1)$, then, by Lemma 13(a) and (b), the integral in the right-hand side of (79) belongs to $C_{\mu-\alpha, \log }[a, b]$ for $\mu>\alpha$ and to $C[a, b]$ for $\mu \leq \alpha$. Since $\mu-\alpha<1-\gamma$, by Lemma 16 the right-hand side of (79) belongs to $C_{1-\gamma, \log }[a, b]$.

Our second claim is that $T$ is a contraction; that is,

$$
\begin{array}{r}
\left\|T y_{1}-T y_{2}\right\|_{C_{1-\gamma, \log }\left[a, x_{1}\right]} \leq w_{1}\left\|y_{1}-y_{2}\right\|_{C_{1-\gamma, \log }\left[a, x_{1}\right]}, \\
0<w_{1}<1 .
\end{array}
$$

This follows from (79), Lemma 13(a), and the fact that

$$
\begin{aligned}
& \left\|T y_{1}-T y_{2}\right\|_{C_{1-\gamma, \log }\left[a, x_{1}\right]} \\
& =\left\|\mathcal{J}_{a^{+}}^{\alpha} f\left[t, y_{1}(t)\right]-\mathcal{J}_{a^{+}}^{\alpha} f\left[t, y_{2}(t)\right]\right\|_{C_{1-\gamma, \log }\left[a, x_{1}\right]} \\
& \leq\left(\log \frac{x_{1}}{a}\right)^{2 \alpha} \frac{\Gamma(\gamma)}{\Gamma(\alpha+\gamma)}\left\|f\left[t, y_{1}(t)\right]-f\left[t, y_{2}(t)\right]\right\|_{C_{1-\gamma, \log }\left[a, x_{1}\right]} \\
& \leq A\left(\log \frac{x_{1}}{a}\right)^{2 \alpha} \frac{\Gamma(\gamma)}{\Gamma(\alpha+\gamma)}\left\|y_{1}(t)-y_{2}(t)\right\|_{C_{1-\gamma, \log }\left[a, x_{1}\right]} \\
& =w_{1}\left\|y_{1}(t)-y_{2}(t)\right\|_{C_{1-\gamma, \log }\left[a, x_{1}\right]} .
\end{aligned}
$$

Our assumption (76) allows us to apply the Banach fixed point theorem to obtain a unique solution $y^{*} \in C_{1-\gamma, \log }\left[a, x_{1}\right]$ to (50) on the interval $\left(a, x_{1}\right]$.

This solution $y^{*}$ is the limit of a convergent sequence $T^{m} y_{0}^{*}$ :

$$
\lim _{m \rightarrow \infty}\left\|T^{m} y_{0}^{*}-y^{*}\right\|_{C_{1-\gamma, \log }\left[a, x_{1}\right]}=0,
$$

where $y_{0}^{*}$ is any function in $C_{1-\gamma, \log }\left[a, x_{1}\right]$ and

$$
\begin{aligned}
\left(T^{m} y_{0}^{*}\right)(x) & =\left(T T^{m-1} y_{0}^{*}\right)(x) \\
& =y_{0}(x)+\left(\mathcal{J}_{a^{+}}^{\alpha} f\left[t,\left(T^{m-1} y_{0}^{*}\right)(t)\right]\right)(x), \\
& m \in \mathbf{N} .
\end{aligned}
$$

Let us take $y_{0}^{*}(x)=y_{0}(x)$ with $y_{0}(x)$ defined by (80). If we denote by

$$
y_{m}(x):=\left(T^{m} y_{0}^{*}\right)(x), \quad m \in \mathbf{N}
$$


then, clearly,

$$
\lim _{m \rightarrow \infty}\left\|y_{m}-y^{*}\right\|_{C_{1-\gamma, \log }\left[a, x_{1}\right]}=0
$$

Next, we consider the interval $\left[x_{1}, b\right]$. From (50) we have

$$
\begin{aligned}
y(x)= & \frac{c}{\Gamma(\gamma)}\left(\log \frac{x}{a}\right)^{\gamma-1} \\
& +\frac{1}{\Gamma(\alpha)} \int_{a}^{x_{1}}\left(\log \frac{x}{t}\right)^{\alpha-1} \frac{f[t, y(t)]}{t} d t \\
& +\frac{1}{\Gamma(\alpha)} \int_{x_{1}}^{x}\left(\log \frac{x}{t}\right)^{\alpha-1} \frac{f[t, y(t)]}{t} d t \\
= & y_{01}(x)+\frac{1}{\Gamma(\alpha)} \int_{x_{1}}^{x}\left(\log \frac{x}{t}\right)^{\alpha-1} \frac{f[t, y(t)]}{t} d t
\end{aligned}
$$

where $y_{01}(x)$ is defined by

$$
\begin{aligned}
y_{01}(x)= & \frac{c}{\Gamma(\gamma)}\left(\log \frac{x}{a}\right)^{\gamma-1} \\
& +\frac{1}{\Gamma(\alpha)} \int_{a}^{x_{1}}\left(\log \frac{x}{t}\right)^{\alpha-1} \frac{f[t, y(t)]}{t} d t,
\end{aligned}
$$

and is a known function. We note that $y_{01} \in C\left[x_{1}, b\right]$.

We want to prove the existence of a unique solution $y \in$ $C\left[x_{1}, b\right]$ of $(50)$ on the interval $\left[x_{1}, b\right]$. For this, we also use Banach fixed point theorem for the space $C\left[x_{1}, x_{2}\right]$ where $x_{2} \in\left(x_{1}, b\right]$ satisfies

$$
w_{2}=\frac{A}{\alpha \Gamma(\alpha)}\left(\log \frac{x_{2}}{x_{1}}\right)^{\alpha}<1
$$

The space $C\left[x_{1}, x_{2}\right]$ is a complete metric space with the distance given by

$$
d\left(y_{1}, y_{2}\right)=\left\|y_{1}-y_{2}\right\|_{C\left[x_{1}, x_{2}\right]}=\max _{x \in\left[x_{1}, x_{2}\right]}\left|y_{1}(x)-y_{2}(x)\right|
$$

The integral equation (87) may be written shortly as

$$
y(x)=(T y)(x)
$$

where the operator (again denoted by $T$ ) is given by

$$
(T y)(x)=y_{01}(x)+\frac{1}{\Gamma(\alpha)} \int_{x_{1}}^{x}\left(\log \frac{x}{t}\right)^{\alpha-1} \frac{f[t, y(t)]}{t} d t
$$

As in the first part of this proof, since $y_{01} \in C\left[x_{1}, x_{2}\right]$ and $f[\cdot, y(\cdot)] \in C_{\mu, \log }[a, b]$ for any $y \in C_{\mu, \log }[a, b]$, then, $f[\cdot$, $y(\cdot)] \in C\left[x_{1}, x_{2}\right]$, for any $y \in C\left[x_{1}, x_{2}\right]$, then by Lemma 14 , we deduce that the integral in the right-hand side of (92) also belongs to $C\left[x_{1}, x_{2}\right]$, and hence $T y \in C\left[x_{1}, x_{2}\right]$.
Moreover, using the Lipschitz condition and applying the Lemma 14, we find

$$
\begin{aligned}
& \left\|T y_{1}-T y_{2}\right\|_{C\left[x_{1}, x_{2}\right]} \\
& \quad=\left\|\mathscr{F}_{x_{1}^{+}}^{\alpha} f\left[t, y_{1}(t)\right]-\mathcal{J}_{x_{1}^{+}}^{\alpha} f\left[t, y_{2}(t)\right]\right\|_{C\left[x_{1}, x_{2}\right]} \\
& \leq \frac{1}{\alpha \Gamma(\alpha)}\left(\log \frac{x_{2}}{x_{1}}\right)^{\alpha}\left\|f\left[t, y_{1}(t)\right]-f\left[t, y_{2}(t)\right]\right\|_{C\left[x_{1}, x_{2}\right]} \\
& \leq \frac{A}{\alpha \Gamma(\alpha)}\left(\log \frac{x_{2}}{x_{1}}\right)^{\alpha}\left\|y_{1}(t)-y_{2}(t)\right\|_{C\left[x_{1}, x_{2}\right]} \\
& \quad=w_{2}\left\|y_{1}(t)-y_{2}(t)\right\|_{C\left[x_{1}, x_{2}\right]} .
\end{aligned}
$$

This, together with our assumption $0<w_{2}<1$, shows that $T$ is a contraction and therefore from Theorem 17, there exists a unique solution $y_{1}^{*} \in C\left[x_{1}, x_{2}\right]$ to (50) on the interval $\left[x_{1}, x_{2}\right]$. Notice that $y_{1}^{*}\left(x_{1}\right)=y^{*}\left(x_{1}\right)=y_{01}\left(x_{1}\right)$. Further, Theorem 17 guarantees that this solution is the limit of a convergent sequence $T^{m} y_{01}^{*}$ :

$$
\lim _{m \rightarrow \infty}\left\|T^{m} y_{01}^{*}-y_{1}^{*}\right\|_{C\left[x_{1}, x_{2}\right]}=0
$$

where $y_{01}^{*}$ is any function in $C\left[x_{1}, x_{2}\right]$, which we can pick $y_{01}^{*}(x)=y_{01}(x)$ defined by $(88)$. Therefore,

$$
\lim _{m \rightarrow \infty}\left\|y_{m}-y_{1}^{*}\right\|_{C\left[x_{1}, x_{2}\right]}=0,
$$

where

$$
\begin{aligned}
y_{m}(x)= & \left(T^{m} y_{01}^{*}\right)(x) \\
= & y_{01}(x)+\frac{1}{\Gamma(\alpha)} \\
& \times \int_{x_{1}}^{x}\left(\log \frac{x}{t}\right)^{\alpha-1} \frac{f\left[t,\left(T^{m-1} y_{01}^{*}\right)(t)\right]}{t} d t .
\end{aligned}
$$

If $x_{2} \neq b$, we consider the interval $\left[x_{2}, x_{3}\right]$, where $x_{3}=x_{2}+h_{2}$, $h_{2}>0$ such that $x_{3} \leq b$ and

$$
w_{3}=\frac{A}{\alpha \Gamma(\alpha)}\left(\log \frac{x_{3}}{x_{2}}\right)^{\alpha}<1 .
$$

Using the same arguments as above, we derive that there exists a unique solution $y_{2}^{*} \in C\left[x_{2}, x_{3}\right]$ to (50) on the interval $\left[x_{2}, x_{3}\right]$. If $x_{3} \neq b$, then we continue the process until we reach a solution $y$ to (50), $y(x)=y_{k}^{*}(x)$, and $y_{k}^{*} \in C\left[x_{k}, x_{k+1}\right]$ $(k=1, \ldots, L)$, where $a=x_{0}<x_{1}<\cdots<x_{L+1}$ and

$$
w_{k+1}=\frac{A}{\alpha \Gamma(\alpha)}\left(\log \frac{x_{k+1}}{x_{k}}\right)^{\alpha}<1 .
$$

Assume that $b-a>\Gamma(\alpha+\gamma) / A \Gamma(\gamma)$ (for otherwise, take $\left.x_{1}=b\right)$. Then, divide the length of the interval $[a+\Gamma(\alpha+$ $\gamma) / A \Gamma(\gamma), b]$ by $(\alpha \Gamma(\alpha) / A)^{1 / \alpha}$. Let $M$ be that quotient. It appears that for $L=[M]+1$ and $b$ is reached after a finite number of steps, $x_{L+1}=b$. Then, there exists a unique solution $y \in C\left[x_{1}, b\right]$ to $(50)$ on the interval $\left[x_{1}, b\right]$. 
Putting together the solutions in $\left[a, x_{1}\right]$ and $\left[x_{1}, b\right]$ and taking into account the Lemma 15, we obtain that there exists a unique solution $y \in C_{1-\gamma, \log }[a, b]$ to the Volterra integral equation (50) on the whole interval $(a, b]$. Hence, $y \in C_{1-\gamma, \log }[a, b]$ is the unique solution to the Cauchy-type problem (46).

It remains to show that such a unique solution is actually in $C_{1-\gamma, \mu}^{\alpha, \beta}[a, b]$. To this end we need to prove that ${ }_{H^{2}} \mathscr{D}_{a^{+}}^{\alpha, \beta} y \in$ $C_{\mu, \log }[a, b]$. Let us recall that our $y$ is a limit of the sequence $y_{m}$, where $y_{m}=T^{m} y_{0}^{*} \in C_{1-\gamma, \log }[a, b]$; that is,

$$
\lim _{m \rightarrow \infty}\left\|y_{m}-y\right\|_{C_{1-\gamma, \log }[a, b]}=0 \text {, }
$$

with a certain choice of $y_{0}^{*}(x)$ on each subinterval $\left[a, x_{1}\right]$, $\ldots,\left[x_{L}, b\right]$. Indeed, this is a consequence of the construction adopted, the initial values are selected in the space $C_{1-\gamma, \log }[a, b]$ (see $(80)$ and $(88)$ ) and the operator maps this space into itself (see argument right after (80) and (92)). As for the convergence in that space it has been proved in (83) and (95).

If $y_{0}(x) \neq 0$, then we can take $y_{0}^{*}(x)=y_{0}(x)$. Since $\mu \geq$ $1-\gamma$, then by (46), the Lipschitz condition and Lemma 8 , we have

$$
\begin{aligned}
\| H^{\mathscr{D}_{a^{+}}^{\alpha, \beta}} y_{m}-H^{2} & \mathscr{D}_{a^{+}}^{\alpha, \beta} y \|_{C_{\mu, \log }[a, b]} \\
& =\left\|f\left[x, y_{m}\right]-f[x, y]\right\|_{C_{\mu, \log }[a, b]} \\
& \leq A\left(\log \frac{b}{a}\right)^{\mu-1+\gamma}\left\|y_{m}-y\right\|_{C_{1-\gamma, \log }[a, b]} .
\end{aligned}
$$

In virtue of (99) and (100), it follows that

$$
\lim _{m \rightarrow \infty}\left\|{ }_{H} \mathscr{D}_{a^{+}}^{\alpha, \beta} y_{m}-{ }_{H} \mathscr{D}_{a^{+}}^{\alpha, \beta} y\right\|_{C_{\mu, \log }[a, b]}=0 .
$$

We entail from this relation that $\left({ }_{H} \mathscr{D}_{a^{+}}^{\alpha, \beta} y\right) \in C_{\mu, \log }[a, b]$ if $\left({ }_{H} \mathscr{D}_{a^{+}}^{\alpha, \beta} y_{m}\right) \in C_{\mu, \log }[a, b], m=1,2, \ldots$ This latter property holds in as much as ${ }_{H} \mathscr{D}_{a^{+}}^{\alpha, \beta} y_{m}(x)=f\left[x, y_{m-1}(x)\right]$ and $f[\cdot, y(\cdot)] \in C_{\mu, \log }[a, b]$ for any $y \in C_{\mu, \log }[a, b]$.

Consequently, $y \in C_{1-\gamma, \mu}^{\alpha, \beta}[a, b]$. This completes the proof of Theorem 22.

\section{Stability}

In this section, we consider the weighted Cauchy-type problem

$$
\begin{aligned}
& H_{a^{+}}^{\alpha, \beta} u(t)=f(t, u(t)), \quad t>a>0, \\
& \left.\left(\log \frac{t}{a}\right)^{(1-\beta)(1-\alpha)} u(t)\right|_{t=a}=b,
\end{aligned}
$$

where ${ }_{H} \mathscr{D}_{+}^{\alpha, \beta}$ is the Hilfer-Hadamard fractional derivative (HHFD) of order $0<\alpha<1$ and type $0 \leq \beta \leq 1$ and $b \in \mathbf{R}^{*}$ (the set of all real numbers except 0 ). It is interesting to note that, using an argument similar to the one in the proof of
Lemma 3.5 in [2] (see also Lemma 3.2), we can prove that the initial condition in (102) and the one in (46) are equivalent.

We will assume the following hypotheses on the function $f:$

$\left(\mathbf{F}^{*}\right) f(t, u)$ is a continuous (nonlinear) function on $(a, \infty) \times \mathbf{R}$ and is such that

$$
|f(t, u(t))| \leq\left(\log \frac{t}{a}\right)^{\mu} \varphi(t)|u(t)|^{m}, \quad \mu \geq 0, m>1, t \geq a,
$$

where $\varphi$ is a continuous (nonnegative) function on $[a, \infty)$.

We first prove the following inequality.

Lemma 23. If $\lambda, v, \omega>0$, then for anyt $>a, a>0$ we have

$$
\left(\log \frac{t}{a}\right)^{1-\nu} \int_{a}^{t}\left(\log \frac{t}{s}\right)^{\nu-1}\left(\log \frac{s}{a}\right)^{\lambda-1}\left(\frac{s}{a}\right)^{-\omega} \frac{d s}{s} \leq C \omega^{-\lambda},
$$

where $C$ is a positive constant independent of $t$.

Proof. Let us denote by $I(t)$ the left-hand side of the inequality in the Lemma. We consider the change of variable, $\xi=$ $(\log (s / a)) /(\log (t / a))$ then $s / a=(t / a)^{\xi}$ and $\log (t / s)=(1-$ $\xi)(\log (t / a))$. It follows that

$$
I(t)=\left(\log \frac{t}{a}\right)^{\lambda} \int_{0}^{1}(1-\xi)^{\nu-1} \xi^{\lambda-1}\left(\frac{t}{a}\right)^{-\omega \xi} d \xi,
$$

or

$$
I(t)=\left(\log \frac{t}{a}\right)^{\lambda} \int_{0}^{1}(1-\xi)^{\nu-1} \xi^{\lambda-1} \exp \left(-\omega \xi \log \left(\frac{t}{a}\right)\right) d \xi .
$$

Observe that, for $\xi \geq 1$ and $[\lambda]+1 \geq \lambda$ we have $\xi^{[\lambda]+1} \geq$ $\xi^{\lambda}$. Also since $\lambda+2 \geq[\lambda]+2$ and the Gamma function is increasing in $[2, \infty)$ we have $\Gamma(\lambda+2) \geq \Gamma([\lambda]+2)$ or $1 / \Gamma([\lambda]+$ $2) \geq 1 / \Gamma(\lambda+2)$. Moreover, $e^{\xi} \geq\left(\xi^{[\lambda]+1} / \Gamma([\lambda]+2)\right)(\Gamma([\lambda]+2)=$ $([\lambda]+1) !)$, and hence

$$
e^{\xi} \geq \frac{\xi^{[\lambda]+1}}{\Gamma([\lambda]+2)} \geq \frac{\xi^{\lambda}}{\Gamma([\lambda]+2)} \geq \frac{\xi^{\lambda}}{\Gamma(\lambda+2)},
$$

or

$$
e^{-\xi} \leq \frac{\Gamma(\lambda+2)}{\xi^{\lambda}}
$$

Therefore, for $0 \leq \xi<1 / 2$ we get

$$
(1-\xi)^{\nu-1} \leq \max \left(1,2^{1-\gamma}\right) .
$$

For $1 / 2<\xi \leq 1$ and $t$ such that $\omega \xi \log (t / a) \geq 1$ we have

$$
\begin{aligned}
\exp \left(-\omega \xi \log \left(\frac{t}{a}\right)\right) & \leq \frac{\Gamma(\lambda+2)}{(\omega \xi \log (t / a))^{\lambda}} \leq \frac{\omega^{-\lambda}}{\xi} \Gamma(\lambda+2) \\
& \leq 2 \omega^{-\lambda} \Gamma(\lambda+2)
\end{aligned}
$$


This means that

$$
\begin{aligned}
& \left(\log \frac{t}{a}\right)^{\lambda}(1-\xi)^{\nu-1} \xi^{\lambda-1} \exp \left(-\omega \xi \log \left(\frac{t}{a}\right)\right) \\
& \quad \leq\left\{\begin{array}{cl}
\max \left(1,2^{1-\nu}\right)\left(\log \frac{t}{a}\right)^{\lambda} \xi^{\lambda-1} \\
\times \exp \left(-\omega \xi \log \left(\frac{t}{a}\right)\right), & 0 \leq \xi<\frac{1}{2}, \\
2(1-\xi)^{\nu-1} \Gamma(\lambda+2) \omega^{-\lambda}, & \frac{1}{2}<\xi \leq 1 .
\end{array}\right.
\end{aligned}
$$

Consequently,

$$
\begin{aligned}
I(t) \leq & \max \left(1,2^{1-\nu}\right)\left(\log \frac{t}{a}\right)^{\lambda} \\
& \times \int_{0}^{1 / 2} \xi^{\lambda-1} \exp \left(-\omega \xi \log \left(\frac{t}{a}\right)\right) d \xi \\
& +2 \omega^{-\lambda} \Gamma(\lambda+2) \int_{1 / 2}^{1}(1-\xi)^{\nu-1} d \xi
\end{aligned}
$$

Let $u=\omega \xi \log (t / a)$, we see that

$$
\begin{aligned}
I(t) \leq & \max \left(1,2^{1-\nu}\right)\left(\log \frac{t}{a}\right)^{\lambda} \\
& \times \int_{0}^{\infty}\left(\frac{u}{\omega \log (t / a)}\right)^{\lambda-1} e^{-u} \frac{d u}{\omega \log (t / a)} \\
& +2 \omega^{-\lambda} \Gamma(\lambda+2)\left[-\frac{(1-\xi)^{\nu}}{\nu}\right]_{\xi=1 / 2}^{1} .
\end{aligned}
$$

Thus,

$$
I(t) \leq \max \left(1,2^{1-\nu}\right) \omega^{-\lambda} \Gamma(\lambda)+\frac{2^{1-\nu} \omega^{-\lambda} \Gamma(\lambda+2)}{\nu} .
$$

As a result, $I(t) \leq \max \left\{1,2^{1-\nu}\right\} \Gamma(\lambda)(1+\lambda(\lambda+1) / \nu) \omega^{-\lambda}$.

For $0<\eta<1, e^{\eta} \geq 1$ it is clear that

$$
\Gamma(\lambda+2) e^{\eta} \geq 1 \geq \eta^{\lambda}
$$

holds and we proceed in the same manner to conclude that for $t$ such that $0<\omega \xi \log (t / a)<1$

$$
\left(\log \frac{t}{a}\right)^{1-\nu} \int_{a}^{t}\left(\log \frac{t}{s}\right)^{\nu-1}\left(\log \frac{s}{a}\right)^{\lambda-1}\left(\frac{s}{a}\right)^{-\omega} \frac{d s}{s} \leq C \omega^{-\lambda},
$$

where $C=\max \left\{1,2^{1-\nu}\right\} \Gamma(\lambda)(1+\lambda(\lambda+1) / \nu)$. The proof is complete.

Let $p$ and $q$ be conjugate exponents; that is, $p q=p+q$, and let $\lambda_{1}:=1+p[\mu-(1-\gamma) m]$ and $\lambda_{2}:=1+p(\alpha-1)$, where $\gamma:=\alpha+\beta-\alpha \beta$. If $\mu-(m-1)(1-\gamma)>0$ and $q>1 / \alpha$, then $\lambda_{1}>0$ and $\lambda_{2}>0$. We denote by $\mathbf{L}^{*}$ the positive real number

$$
\begin{aligned}
\mathbf{L}^{*}:= & \left(\frac{\Gamma(\alpha)}{2^{m+(\alpha-1)}|b|^{m-1}}\right)^{m}\left(\frac{(2 a)^{m}}{m-1}\right)^{1 / q} \\
& \times\left[\frac{(p-1)^{\lambda_{1}}}{\Gamma\left(\lambda_{1}\right)\left(1+\lambda_{1} / \lambda_{2}\right)}\right]^{m / p} .
\end{aligned}
$$

Theorem 24. Suppose that $f$ satisfies $\left(\mathbf{F}^{*}\right)$ and $\mu>(m-1)(1-$ $\gamma)$. If

$$
\left(\|\varphi(t)\|_{q}\right)^{m-1}\left\|\varphi(t)\left(\log \frac{t}{a}\right)^{-m \beta(1-\alpha)}\right\|_{q}<\mathbf{L}^{*}
$$

for some $q>1 / \alpha$, then, for any solution of Problem (102), there exists a positive constant $C$ such that $|u(t)| \leq C(\log (t / a))^{\gamma-1}$, $t>a, a>0$, where $\gamma=\alpha+\beta-\alpha \beta$.

Proof. Let us consider the Volterra integral equation

$$
\begin{array}{r}
u(t)=b\left(\log \frac{t}{a}\right)^{\gamma-1}+\frac{1}{\Gamma(\alpha)} \int_{a}^{t}\left(\log \frac{t}{s}\right)^{\alpha-1} f[s, u(s)] \frac{d s}{s} \\
t>a>0
\end{array}
$$

associated to problem (102). Multiplying both sides of (119) by $(\log (t / a))^{1-\gamma}$ and using the assumption $\left(\mathbf{F}^{*}\right)$ on $f$ we get

$$
\begin{aligned}
& \left(\log \frac{t}{a}\right)^{1-\gamma}|u(t)| \\
& \leq|b|+\frac{(\log (t / a))^{1-\gamma}}{\Gamma(\alpha)} \\
& \quad \times \int_{a}^{t}\left(\log \frac{t}{s}\right)^{\alpha-1}\left(\log \frac{s}{a}\right)^{\mu} \varphi(s)|u(s)|^{m} \frac{d s}{s} .
\end{aligned}
$$

Let $v(t)$ denote the left-hand side of (120). The insertion of the term

$$
\left(\log \frac{s}{a}\right)^{(1-\gamma) m}\left(\log \frac{s}{a}\right)^{-(1-\gamma) m}
$$

inside the integral gives

$$
\begin{aligned}
& v(t) \leq|b|+\frac{(\log (t / a))^{1-\gamma}}{\Gamma(\alpha)} \\
& \times \int_{a}^{t}\left(\log \frac{t}{s}\right)^{\alpha-1}\left(\log \frac{s}{a}\right)^{\mu-(1-\gamma) m} \\
& \quad \times \varphi(s) v^{m}(s) \frac{d s}{s}, \quad t>a .
\end{aligned}
$$

Now, the Hölder inequality with exponents $p$ and $q$ yields

$$
\begin{aligned}
& \int_{a}^{t}\left(\log \frac{t}{s}\right)^{\alpha-1}\left(\log \frac{s}{a}\right)^{\mu-(1-\gamma) m} \varphi(s) v^{m}(s) \frac{d s}{s} \\
& \leq\left(\int_{a}^{t}\left(\log \frac{t}{s}\right)^{p(\alpha-1)}\left(\log \frac{s}{a}\right)^{p(\mu-(1-\gamma) m)} \frac{d s}{s^{p}}\right)^{1 / p} \\
& \quad \times\left(\int_{a}^{t} \varphi^{q}(s) v^{q m}(s) d s\right)^{1 / q} \\
& \leq a^{-1 / q}\left(\int_{a}^{t}\left(\log \frac{t}{s}\right)^{p(\alpha-1)}\left(\log \frac{s}{a}\right)^{p(\mu-(1-\gamma) m)}\left(\frac{s}{a}\right)^{-(p-1)} \frac{d s}{s}\right)^{1 / p} \\
& \quad \times\left(\int_{a}^{t} \varphi^{q}(s) v^{q m}(s) d s\right)^{1 / q}, \quad t>a .
\end{aligned}
$$


Since $\lambda_{1}-1=p[\mu-(1-\gamma) m], \lambda_{2}-1=p(\alpha-1)$ and $\lambda_{1}, \lambda_{2}$, $p-1>0$, we may apply Lemma 9 (with $\nu$ replaced by $\lambda_{2}, \lambda$ replaced by $\lambda_{1}$ and $\omega$ replaced by $\left.p-1\right)$ to get

$$
\begin{aligned}
\int_{a}^{t}\left(\log \frac{t}{s}\right)^{\alpha-1}\left(\log \frac{s}{a}\right)^{\mu-(1-\gamma) m} \varphi(s) v^{m}(s) \frac{d s}{s} \\
\leq a^{-1 / q} C_{1}\left(\log \frac{t}{a}\right)^{\alpha-1} \\
\quad \times\left(\int_{a}^{t} \varphi^{q}(s) v^{q m}(s) d s\right)^{1 / q}, \quad t>a,
\end{aligned}
$$

where $C_{1}$ is the constant appearing in Lemma 9 corresponding to the present exponents. That is,

$$
C_{1}=\left(2^{p(\alpha-1)} \Gamma\left(\lambda_{1}\right)\left(1+\frac{\lambda_{1}\left(\lambda_{1}+1\right)}{\lambda_{2}}\right)(p-1)^{-\lambda_{1}}\right)^{1 / p} .
$$

Combining (122) and (124) we entail that

$$
\begin{aligned}
v(t) \leq & |b|+\widehat{C}_{1}\left(\log \frac{t}{a}\right)^{-\beta(1-\alpha)} \\
& \times\left(\int_{a}^{t} \varphi^{q}(s) v^{q m}(s) d s\right)^{1 / q}, \quad t>a,
\end{aligned}
$$

where $\widehat{C}_{1}=a^{-1 / q}\left(C_{1} / \Gamma(\alpha)\right)$. Multiplying both sides of (126) by $(\log (t / a))^{\beta(1-\alpha)}$, we obtain

$$
\begin{aligned}
&\left(\log \frac{t}{a}\right)^{\beta(1-\alpha)} v(t) \\
& \leq|b|\left(\log \frac{t}{a}\right)^{\beta(1-\alpha)}+\widehat{C}_{1}\left(\int_{a}^{t} \varphi^{q}(s) v^{q m}(s) d s\right)^{1 / q}, \\
& t>a .
\end{aligned}
$$

Let $z(t)$ denote the left-hand side of (127). The insertion of the term

$$
\left(\log \frac{s}{a}\right)^{-q m \beta(1-\alpha)}\left(\log \frac{s}{a}\right)^{q m \beta(1-\alpha)}
$$

inside the integral gives

$$
\begin{aligned}
z(t) \leq & |b|\left(\log \frac{t}{a}\right)^{\beta(1-\alpha)} \\
& +\widehat{C}_{1}\left(\int_{a}^{t} \varphi^{q}(s)\left(\log \frac{s}{a}\right)^{-q m \beta(1-\alpha)} z^{q m}(s) d s\right)^{1 / q} .
\end{aligned}
$$

Raising both sides of (129) to the power $q$ we get

$$
\begin{aligned}
z^{q}(t) \leq 2^{q-1}\left(|b|^{q}\left(\log \frac{t}{a}\right)^{q \beta(1-\alpha)}\right. & \\
& \left.+\widehat{C}_{1}^{q} \int_{a}^{t} \varphi^{q}(s)\left(\log \frac{s}{a}\right)^{-q m \beta(1-\alpha)} z^{q m}(s) d s\right) .
\end{aligned}
$$

Let us set

$$
w(t)=\widehat{C}_{1}^{q} \int_{a}^{t} \varphi^{q}(s)\left(\log \frac{s}{a}\right)^{-q m \beta(1-\alpha)} z^{q m}(s) d s, \quad t>a .
$$

Then, clearly $w(a)=0$, and by differentiation

$$
w^{\prime}(t)=\widehat{C}_{1}^{q} \varphi^{q}(t)\left(\log \frac{t}{a}\right)^{-q m \beta(1-\alpha)} z^{q m}(t), \quad t>a .
$$

Moreover, it is clear that $w$ is a continuous, nonnegative and nondecreasing function in $[a, \infty)$.

Now, we would like to estimate the right hand side of (132) in term of $w(t)$. From (130) and (131) we entail that

$$
z^{q}(t) \leq 2^{q-1}\left(|b|^{q}\left(\log \frac{t}{a}\right)^{q \beta(1-\alpha)}+w(t)\right), \quad t>a .
$$

Raising both sides of (133) to the power $m$ we get

$$
z^{q m}(t) \leq 2^{m q-1}\left(|b|^{m q}\left(\log \frac{t}{a}\right)^{m q \beta(1-\alpha)}+w^{m}(t)\right), \quad t>a .
$$

The substitution of (134) in (132) yields

$$
\begin{aligned}
w^{\prime}(t) \leq & 2^{m q-1} \widehat{C}_{1}^{q} \varphi^{q}(t)\left(\log \frac{t}{a}\right)^{-q m \beta(1-\alpha)} \\
& \times\left(|b|^{m q}\left(\log \frac{t}{a}\right)^{m q \beta(1-\alpha)}+w^{m}(t)\right) \\
\leq & 2^{m q-1}|b|^{m q} \widehat{C}_{1}^{q} \varphi^{q}(t)+2^{m q-1} \widehat{C}_{1}^{q} \\
& \times\left(\log \frac{t}{a}\right)^{-q m \beta(1-\alpha)} \varphi^{q}(t) w^{m}(t) .
\end{aligned}
$$

Applying Lemma $16\left(\right.$ with $\left.w(u)=u^{m}\right)$ we infer that

$$
\begin{aligned}
w(t) \leq G^{-1}[ & G\left(w(a)+2^{m q-1}|b|^{m q} \widehat{C}_{1}^{q} \int_{a}^{t} \varphi^{q}(s) d s\right) \\
& \left.+2^{m q-1} \widehat{C}_{1}^{q} \int_{a}^{t}\left(\log \frac{s}{a}\right)^{-q m \beta(1-\alpha)} \varphi^{q}(s) d s\right] .
\end{aligned}
$$

Let us set

$$
\begin{aligned}
& l(t)=2^{m q-1}|b|^{m q} \widehat{C}_{1}^{q} \int_{a}^{t} \varphi^{q}(s) d s, \\
& k(t)=2^{m q-1} \widehat{C}_{1}^{q} \int_{a}^{t}\left(\log \frac{s}{a}\right)^{-q m \beta(1-\alpha)} \varphi^{q}(s) d s,
\end{aligned}
$$

then

$$
w(t) \leq G^{-1}[G(l(t))+k(t)],
$$

where we have used the fact that $w(a)=0$. Since $G(r)=$ $\int_{r_{0}}^{r}\left(d s / s^{m}\right), r>0, r_{0}>0$, then $G(r)=\left(r^{1-m} /(1-m)\right)-$ 
$\left(r_{0}^{1-m} /(1-m)\right)$ and $G^{-1}(y)=\left[r_{0}^{1-m}-(m-1) y\right]^{-1 /(m-1)}$. That

is,

$$
\begin{aligned}
& w(t) \leq G^{-1}\left[\frac{l(t)^{1-m}}{1-m}-\frac{l\left(t_{0}\right)^{1-m}}{1-m}+k(t)\right] \\
& \leq\left[l\left(t_{0}\right)^{1-m}-(m-1)\left(\frac{l(t)^{1-m}}{1-m}-\frac{l\left(t_{0}\right)^{1-m}}{1-m}+k(t)\right)\right]^{-1 /(m-1)} \\
& \leq\left[l(t)^{1-m}-(m-1) k(t)\right]^{-1 /(m-1)} .
\end{aligned}
$$

As long as

$$
l(t)^{m-1} k(t)<\frac{1}{m-1}
$$

In particular, if $\left(\|\varphi(t)\|_{q}\right)^{m-1}\left\|\varphi(t)(\log (t / a))^{-m \beta(1-\alpha)}\right\|_{q}<\mathbf{L}^{*} / \mathbf{2}$, then $w(t) \leq K_{1}$ for some positive constant $K_{1}$ for all $t>a$, and thus, from (129), we see that

$$
z(t) \leq|b|\left(\log \frac{t}{a}\right)^{\beta(1-\alpha)}+K_{1}^{1 / q}
$$

and then

$$
v(t) \leq|b|+K_{1}^{1 / q}\left(\log \frac{t}{a}\right)^{-\beta(1-\alpha)} \leq C, \quad t \geq t_{0}>a
$$

for some positive constant $C$. This yields that $|u(t)| \leq$ $C(\log (t / a))^{\gamma-1}$ for $t \geq t_{0}>a$. The proof is complete.

\section{Acknowledgment}

The authors are very grateful for the financial support and the facilities provided by King Fahd University of Petroleum and Minerals through Project no. 101003.

\section{References}

[1] A. A. Kilbas, B. Bonilla, and J. J. Trujillo, "Fractional integrals and derivatives and differential equations of fractional order in weighted spaces of continuous functions," Doklady of the National Academy of Sciences of Belarus, vol. 44, no. 6, 2000 (Russian).

[2] A. A. Kilbas, H. M. Srivastava, and J. J. Trujillo, "Theory and applications of fractional differential equations," J. Van Mill, Ed., vol. 204 of North-Holland Mathematics Studies, Elsevier, Amsterdam, The Netherlands, 2006.

[3] A. A. Kilbas and A. A. Titioura, "Nonlinear differential equations with Marchaud-Hadamard-type fractional derivative in the weighted space of summable functions," Mathematical Modelling and Analysis, vol. 12, no. 3, pp. 343-356, 2007.

[4] I. Podlubny, Fractional Differential Equations, vol. 198 of Mathematics in Science and Engineering, Academic Press, San Diego, Calif, USA, 1999.

[5] S. G. Samko, A. A. Kilbas, and O. I. Marichev, Fractional Integrals and Derivatives: Theory and Applications, Gordon and Breach, New York, NY, USA, 1993.
[6] M. D. Qassim, K. M. Furati, and N.-e. Tatar, "On a differential equation involving Hilfer-Hadamard fractional derivative," Abstract and Applied Analysis, vol. 2012, Article ID 391062, 17 pages, 2012.

[7] R. Hilfer, "Fractional time evolution," in Applications of Fractional Calculus in Physics, pp. 87-130, World Scientific, London, UK, 2000.

[8] S. Abbas, "Existence of solutions to fractional order ordinary and delay differential equations and applications," Electronic Journal of Differential Equations, vol. 2011, no. 9, pp. 1-11, 2011.

[9] B. Ahmad and S. Sivasundaram, "Some existence results for fractional integro-differential equations with nonlinear conditions," Communications in Applied Analysis, vol. 12, no. 2, pp. 107-112, 2008.

[10] B. Ahmad and S. Sivasundaram, "Some basic results for fractional functional integro-differential equations," Communications in Applied Analysis, vol. 12, no. 4, pp. 467-478, 2008.

[11] K. M. Furati and N.-e. Tatar, "An existence result for a nonlocal fractional differential problem," Fractional Calculus and Applied Analysis Journal, vol. 26, pp. 43-51, 2004.

[12] K. M. Furati and N.-e. Tatar, "Long time behavior for a nonlinear fractional model," Journal of Mathematical Analysis and Applications, vol. 332, no. 1, pp. 441-454, 2007.

[13] S. Hamani, M. Benchohra, and J. R. Graef, "Existence results for boundary-value problems with nonlinear fractional differential inclusions and integral conditions," Electronic Journal of Differential Equations, vol. 2010, no. 20, pp. 1-16, 2010.

[14] A. A. Kilbas, B. Bonilla, and J. J. Trujillo, "Existence and uniqueness theorems for nonlinear fractional differential equations," Demonstratio Mathematican, vol. 33, no. 3, pp. 583-602, 2000.

[15] A. A. Kilbas and S. A. Marzan, "Cauchy problem for differential equation with Caputo derivative," Fractional Calculus and Applied Analysis, vol. 7, no. 3, pp. 297-320, 2004.

[16] A. A. Kilbas and S. A. Marzan, "Nonlinear differential equations with the Caputo fractional derivative in the space of continuously differentiable functions," Differential Equations, vol. 41, no. 1, pp. 84-89, 2005.

[17] C. Kou, J. Liu, and Y. Ye, "Existence and uniqueness of solutions for the cauchy-type problems of fractional differential equations," Discrete Dynamics in Nature and Society, vol. 2010, Article ID 142175, 15 pages, 2010.

[18] R. P. Agarwal, M. Benchohra, and S. Hamani, "A survey on existence results for boundary value problems of nonlinear fractional differential equations and inclusions," Acta Applicandae Mathematicae, vol. 109, no. 3, pp. 973-1033, 2010.

[19] S. A. Abd El-Salam and A. M. A. El-Sayed, "On the stability of some fractional-order non-autonomous systems," Electronic Journal of Qualitative Theory of Differential Equations, no. 6, pp. 1-14, 2007.

[20] A. M. A. El-Sayed and S. A. A. El-Salam, "Weighted Cauchytype problem of a functional differ-integral equation," Electronic Journal of Qualitative Theory of Differential Equations, vol. 2007, no. 30, pp. 1-9, 2007.

[21] A. M. A. El-Sayed and A. S. Abd El-Salam, "Solution of weighted cauchy-type problem of a diffre-integral functional equation," The International Journal of Nonlinear Science, vol. 5, no. 3, pp. 281-288, 2008.

[22] K. M. Furati and N.-e. Tatar, "Power-type estimates for a nonlinear fractional differential equation," Nonlinear Analysis, Theory, Methods and Applications, vol. 62, no. 6, pp. 1025-1036, 2005. 
[23] K. M. Furati and N.-e. Tatar, "Behavior of solutions for a weighted Cauchy-type fractional differential problem," Fractional Calculus and Applied Analysis Journal, vol. 28, pp. 23-42, 2005.

[24] B. G. Pachpatte, Inequalities for Differential and Integral Equations, vol. 197 of Mathematics in Science and Engineering, Academic Press, London, UK, 1998.

[25] P. L. Butzer, A. A. Kilbas, and J. J. Trujillo, "Mellin transform analysis and integration by parts for Hadamard-type fractional integrals," Journal of Mathematical Analysis and Applications, vol. 270, no. 1, pp. 1-15, 2002.

[26] A. A. Kilbas, "Hadamard-type fractional calculus," Journal of the Korean Mathematical Society, vol. 38, no. 6, pp. 1191-1204, 2001. 


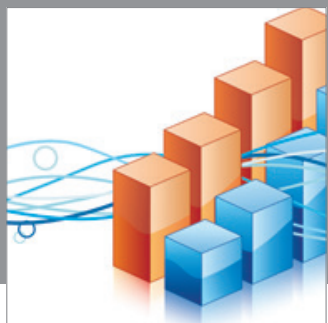

Advances in

Operations Research

mansans

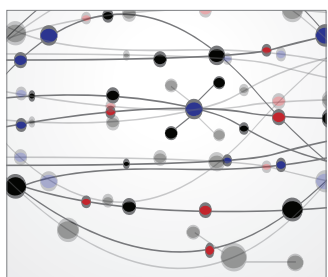

The Scientific World Journal
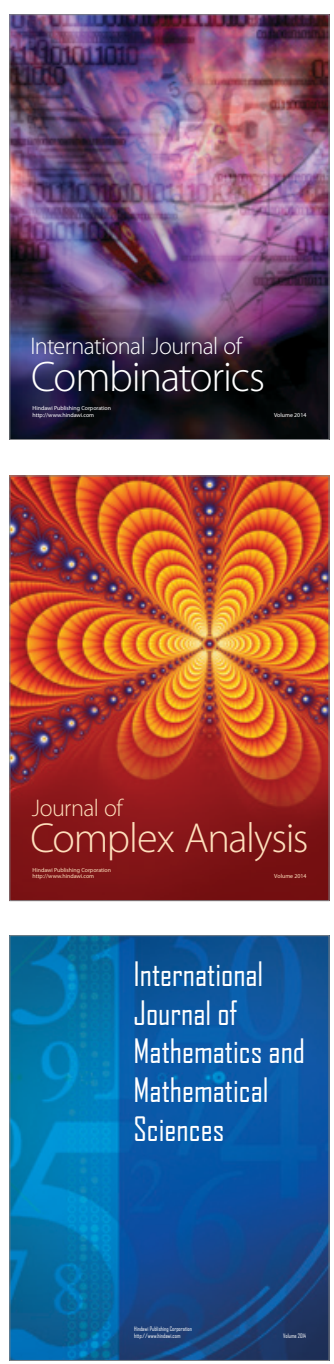
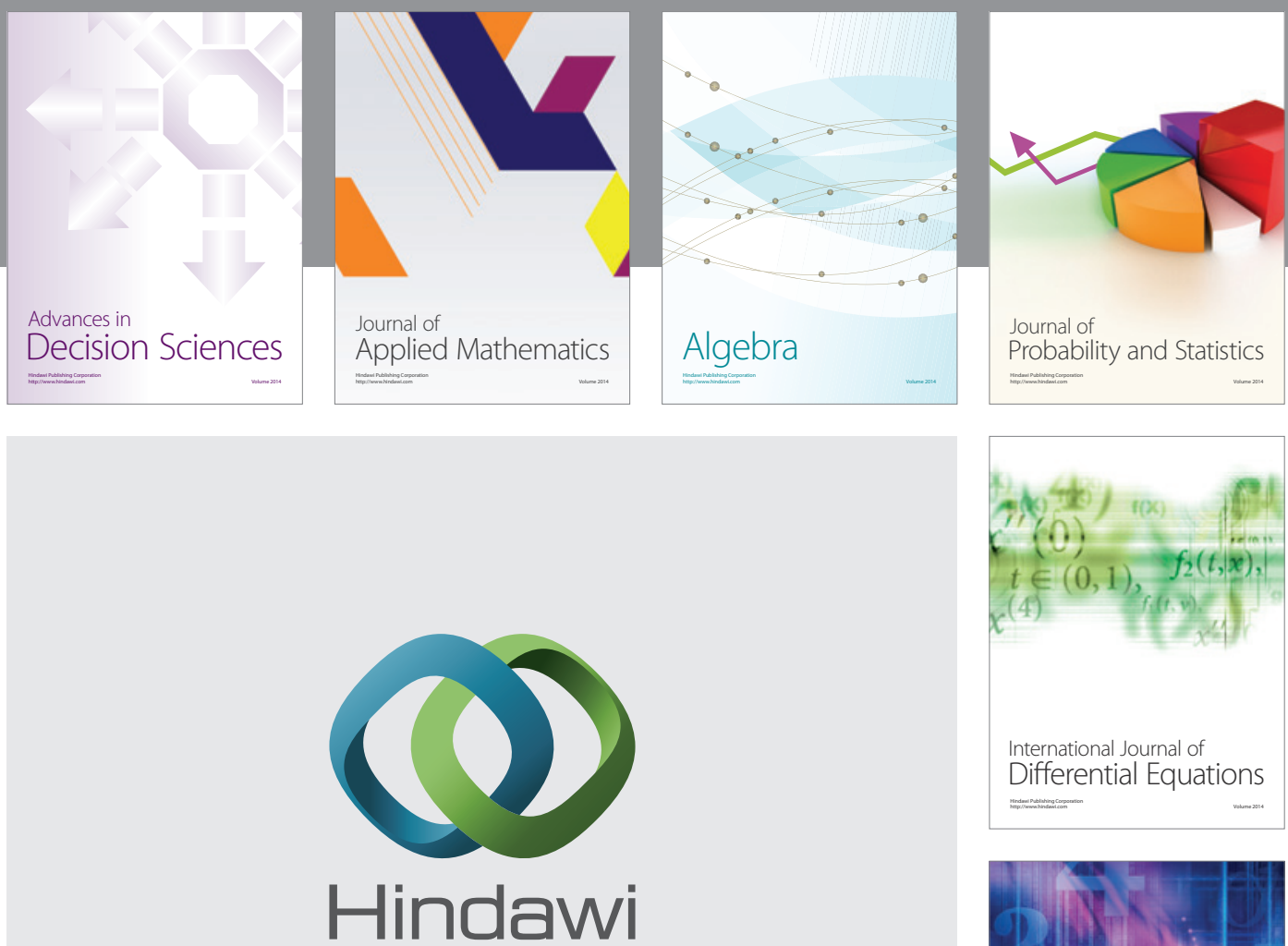

Submit your manuscripts at http://www.hindawi.com
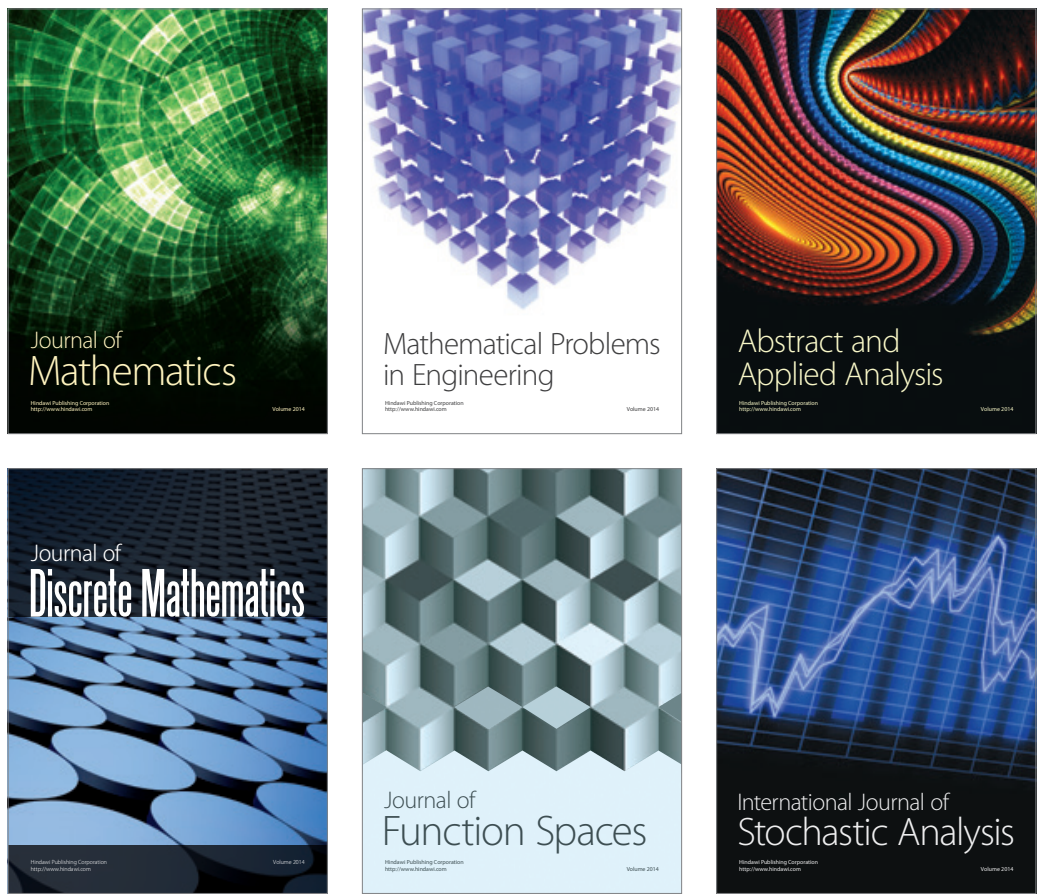

Journal of

Function Spaces

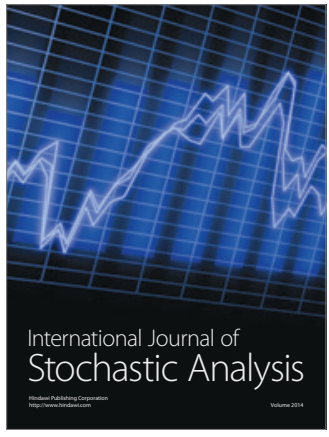

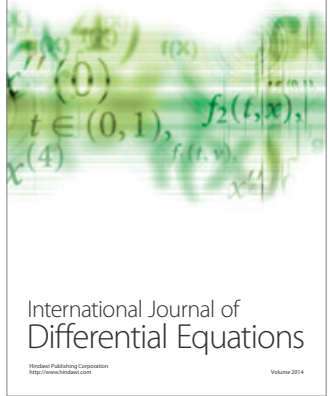
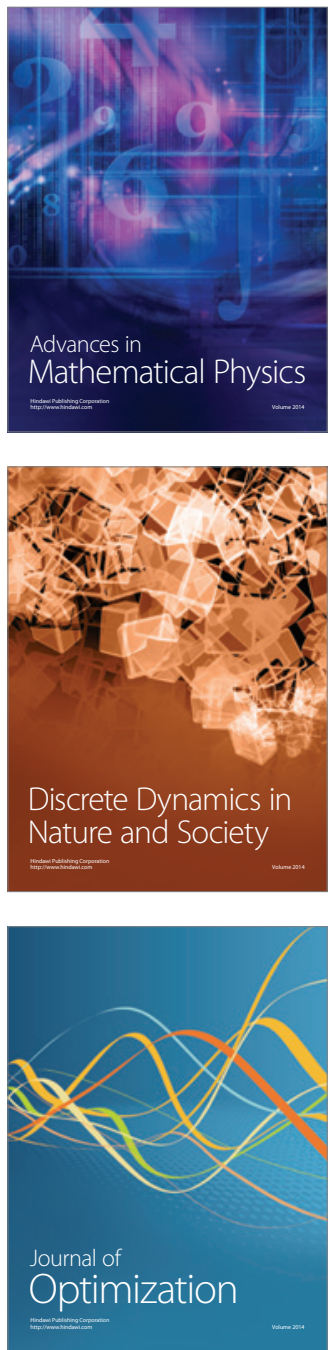